\title{
Considering Communities, Diversity and the Production of Locality in the Design of Networked Urban Screens
}

\author{
Wallis Motta ${ }^{1}$, Ava Fatah gen Schieck ${ }^{1}$, Holger Schnädelbach ${ }^{2}$, \\ Efstathia Kostopoulou ${ }^{1}$, Moritz Behrens ${ }^{1}$, Steve North ${ }^{2}$, and Lei $\mathrm{Ye}^{2}$ \\ ${ }^{1}$ The Bartlett, University College London, UK \\ \{w.motta, ava.fatah, moritz.behrens, ucftefk\}@ucl.ac.uk \\ ${ }^{2}$ Mixed Reality Laboratory (MRL), University of Nottingham, UK \\ \{Holger.Schnadelbach, Steve.North, Lei.Ye\} @nottingham.ac.uk
}

\begin{abstract}
Highly diverse settings such as London (with people from $~ 179$ countries speaking $~ 300$ languages) are unique in that ethnic or socio-cultural backgrounds are no longer sufficient to generate a sense of place, belonging and community. Instead, residents actively perform place building activities on an ongoing basis, which we believe is of great importance when deploying interactive situated technologies in public spaces.

This paper investigates community and place building within a complex multicultural context. We approached this using ethnography, complemented with workshops in the wild. By studying the relationships arising between different segments of the community and two networked screen nodes, we examine the place building activities of residents, and how screen nodes are incorporated into them. Our research suggests that urban screens will be framed (and eventually used) as part of this continuing process of social, spatial and cultural construction. This highlights the importance of enabling socially meaningful relations between the people mediated by these technologies.
\end{abstract}

Keywords: Diversity, communities, ethnography, workshops, in the wild, urban screens.

\section{Introduction}

When studying the deployment of interactive screens in urban public settings [14-17] it is important to consider not only their immediate use and spatial location [2, 13, 17], but also their assimilation within the wider socio-cultural context of the locale. This is of great importance because the interplay between these spatial and cultural elements can strongly influence the long-term success of these technologies. One way in which this interplay can be addressed is by investigating the production of locality $[1,5,18]$. This refers to the processes that communities use to build a sense of place; in other words, how the communities create cultural conventions to control both space and people's behavior within them in ways that allow individuals to develop their own identities in relation to both the locale and other people [1]. By defining who has access to what spaces, at what times, under what conditions and based upon which 
narratives or behaviors, a community can define itself in terms of its conventions, practices, values and aspirations, hence providing a shared social identity.

Place building activities are varied, and they are as much about appropriation as they are about the negotiation and control of space when interacting with other people. For instance, Dant and Deacon observed that homeless people living in hostels emphasized control over what they were allowed to do, such as being able to make a cup of tea whenever they wished, as a way to transform an impersonal space into a place they felt they belonged to [4]. Similarly, some residents in the London neighborhoods where we worked emphasize a degree of control over certain public spaces (e.g. Library halls) to install art exhibitions for public display. In these two cases, certain practical actions (such as making tea or periodically renewing art exhibits) enable social exchanges and conviviality, which translate into a feeling of commonality that underpins the wider social structures that support the community. The analysis of place building is of particular interest in locales where there is a large heterogeneity in ethnicity, nationality, language, religion, educational level and income. In these highly diverse contexts [19] a sense of place does not arise implicitly as a simple product of long held traditions; instead, the local inhabitants must explicitly create it through active efforts of place building [1].

The literature on urban screens has explored the crossroads between communities, public space, technical factors and social interaction around interfaces $[6,10,12,16$, 18]. Although some works address the social aspects of screen use, the study of how wider socio-cultural variables influence long-term community support of urban screens remains an open question. In general, there has been an emphasis on social interactions mediated through screen content on shorter timescales [13, 15, 17], without the consideration of longer term, wider scope socio-cultural variables. Only very few longitudinal case studies have taken a wider contextual approach such as $[14,18]$.

The present paper argues that this wider social context matters if one wants to understand the different perspectives and behaviors surrounding long-term community support for public interactive screens. To study the ways in which such wider cultural context affects screen node use, we explore how urban, networked screens are integrated within the cultural practices of a highly diverse urban locale in London. We examine the social aspects of screen use through an analysis of place building practices, and inquire into the reasons why certain people emphasize certain uses for the screens as opposed to others. Our research takes a targeted, longitudinal approach to understand a complex range of social, technical and interactional issues [2, 6, 12].

Our data gathering methodology includes ethnography and workshops in the wild. The main contribution of this work is to show how action, space and sociality mutually impinge upon one another forming a wider cultural logic, which in turn has a great influence on how a network of public interactive screens is experienced, treated, understood and embraced in practice.

The structure of the paper is as follows. First, we describe our methodology in Section 2. In Section 3 we discuss the integration of screen nodes within pre-existing place building activities. We provide further analysis and conclusions in Section 4. 


\section{Methodology}

Our project involves a network of four interactive touch screens designed to encourage public participation and to explore how networked urban screens can augment urban experience to support communities and culture [6]. There were three main screen node applications discussed with our participants during workshops and ethnography: Slideshow, SoundShape and ScreenGram. Slideshow simply loops through a set of images. SoundShape enables people connected remotely to create a collaborative musical pattern. ScreenGram leverages common technologies (e.g. Twitter) to enable users to upload images to the screen nodes. For further description see [12].

Diversity is a fundamental consideration of this project: two of these screens are located in London, which is home to people from $\sim 179$ countries and speaking 300 languages [19]. Furthermore, the borough where these are located (Waltham Forest) is one of the most ethnically diverse in Britain [22]. One of our screen sites is The Mill in Walthamstow, a non-profit organization that runs a building that has become a hub for local communities; the second one, Leytonstone Public Library, fulfills a similar social role. Amongst many other uses, locals use these two sites to hold business meetings, playgroups and art exhibitions, and also to give and/or take free courses. Both The Mill and Leytonstone Library are popular public places where people organize and enact initiatives that actively build a sense of local identity (a sense of place) for both their neighborhoods and the borough itself. Both sites bring together a diverse cross-section of local residents to pursue varied social activities, and hence represent a natural sample of people present in the locality.

In order to engage with a wide range of social groups within these diverse communities, we carried out ethnography and conducted two workshops in Leytonstone and four in Walthamstow. Rather than defining our groups in terms of statistical or demographic properties, we chose to target "grassroots" groups that were already being sustained through various degrees of involvement with the locality. This enabled us to interact with people in their usual social configurations and preestablished networks of relationships. A total of 70 people from the locality participated in the workshops. These people came from a wide variety of cultural backgrounds and age brackets; with roughly equal numbers of toddlers, children, teenagers, adults and seniors. In addition to the workshops, we also carried out ethnography, consisting of observations during the various stages of implementation for over a year, and conducted 64 semi-structured interviews with locals belonging to different social groups. In all our research encounters we discussed a broad selection of issues that included the locale, the screen, and issues relating to a sense of community and belonging.

Each one of the workshops was built around the activities of one or more local groups with which the researchers established contact after engaging ethnographically with the locale for $\sim 8$ weeks. These groups emerged from social meeting grounds such as churches, local schools, activism societies and hobbyist communities. Each group was chosen to be not only as homogeneous as possible, but also to fit with other groups so that, in aggregate, it would provide as wide a view into the dynamic of the locale as possible. Although we did not specifically create our groups on this basis, as 
the research progressed we found that age provided a convenient means to identify sets of groups which manifested consistent behaviors. Hence, regarding the analysis of our data, we chose to divide the whole population into three age-related groups: children/teenagers, adults/young adults, and seniors. This classification, although mainly intended to facilitate data analysis, is supported by the social dynamics of our sites. For instance, a group of children in the locale that participated in our workshops also participate in playgroups with their parents. Another example of a local group that participated in our workshops is The Recycled Teens, a group of seniors that gathers every Tuesday in a senior residence to sing old tunes, watch old films and discuss current affairs in relation to past events. Of course, some groups are not as homogeneous as these. An example of this kind is a knitting group, that includes participants ranging from children as young as 6 to others over 65 years old. In between creating scarves and hats, they discuss local, national and international politics. They use their knitting to support and draw attention to causes. Also this group participated in one of our workshops. In the following section we discuss how our informants integrated screen nodes within these pre-existing place-building activities. We will follow the three age-centered categories we defined earlier in this section.

\section{Embedding Screen Nodes within Place Building Activities}

\subsection{Screens Approached through Competitive Physical Action}

One of the ways in which children and teens generate a sense of belonging, community and place is by playing in public spaces after school hours. These include playgrounds, parks, markets, and sites like The Mill and Leytonstone Library. It is here where they meet friends that can become an integral part of their life. Discovering and mastering the spatial landscape and its community helps children and teenagers build their personal and social identity, while at the same time, helping them feel their neighborhoods as actually theirs. In a way, children and teens seek to assert certain degree of control over their spaces and their social experience within, eventually claiming these places as an extension of their own selves. To a great extent, this is done through a highly embodied, physical engagement. This means that a park or public space only becomes theirs in action [20]: by wandering around, playing in it, and interacting with friends and family both in the space and with the space.

From both ethnographic observations and workshops we noticed that, when interacting with the screen node, children and teens use the same action-centered approach that they use when they make public spaces theirs. They like to explore the opportunities provided by the technology: the sensitivity of the touch foil, the fluidity in application response, and their ability to exert control over the system. Whether it is by simply moving the pointer or by systematically exploring the possibilities in touch response, their approach is dominated by the actions that they can perform rather than by the explicit interactions that the developer envisaged for the application running in the node. Children and teens hence use the node in novel ways, inventing competitive games that rely on physical interaction (e.g. free pointer motion) and play them with others. In this way they create their own uses for the screen, which are 
quite independent from those intended by the application designers (who in our project imagined mainly collaborative, rather than competitive applications). In this case, users invent competitive games because it is easier for them to embed competition within their playground politics, as compared with the abstract collaboration ideal presented by the application itself. In both of our sites children and young teens attend playgroups where they learn to share the space with others; they enjoy books, toys and resources that are not theirs, but which they can borrow and play with. These objects and spaces are not seen as alien, but as personal, in that "public property may become viewed as private possessions and thereby potentially contribute to a sense of self" [3]. Children frame the screen node using a similar collective ownership approach, and they treat it as personally enjoyable, yet community-owned. For these participants, the screen node is experienced in the same way as a park or a playgroup, and social behavior is enacted accordingly.

\subsection{Screens Used as Springboards for Local Culture}

When constructing a sense of belonging, adults emphasize less the physicality of the environment and take a more instrumental view. They are interested in using the screen node to create what they consider a positive environment by improving education, increasing historical awareness or fostering better communication flows between different groups within their neighborhoods. This place building perspective has resulted in the formation of many of initiatives, from the creation of grass-roots business improvement districts, to art trails and community centers. In this context, generating a "Walthamstow/Leytonstone culture" is relevant to adults as an intellectual, symbolic and material practice that not only enables the production of a sense of place, but also promotes "the human capacity to expand worlds towards other potentially distant horizons and more complex outcomes of life" [11]. For our adult participants, place building is an opportunity for the purposeful pursuit of change in the locality; it is a scaffold [8], an instrument to mediate between their imagined view of their locality and the reality in which it stands. Hence, our adult participants see their place building activities as a springboard to generate local culture and bridge the gap between what their locale is and what it could become; a stage for the display of the unrealized potential development of the locale. For instance, those explicitly engaged in transformative activities (such as local activism) wanted to use it to extend their efforts, informing and educating the local population about relevant projects. Members of the Knitting Group, expressed this view, urging researchers to upload to ScreenGram images of knitted characters they were selling to raise funds for their local community center. Local artists thought that the node could extend the available physical exhibition spaces by imagining it as a window to display digital art. The managers of The Mill imagined the node as a virtual notice board, extending their own functions and enabling users to connect with each other even if the building itself was closed. Even those adults not involved in specific community-building projects identified the node as an opportunity for dissemination of community ideas, particularly as outlets of local useful information: recommendations on what to do in the area, news on what is happening and mechanisms to connect with other locals. 
As shown above, most adults in our workshops and interviews reported that they expected the screen node to embody a higher purpose. They approached the screen as an extension of their pre-existing place building endeavors, uploading photos or short messages that were relevant to their community. For many, the mere physical presence of the node itself legitimized previous efforts for place building, showing them to have been efficacious, as new resources for the realization of the community had been produced. These locals presented the node in local events with pride, as a symbol of their achievements to bring the community closer to its unrealized potential.

\subsection{Screens Used as Collective Memory Fixing Mechanisms}

One important way in which seniors generate a sense of place is through remembrance narratives involving the past of the neighborhood and their lives within it. This activity is one of anchoring [7], in which subjects bring the past to inform the present, and ensure that valuable historical lessons remain with us. Although this is particularly visible when they interact with each other during community group meetings, these narratives are also important in other contexts. Whether sharing these stories with children in local schools or with local historical societies, seniors enjoy talking about the past in public spaces.

Given this predilection for reminiscence, our senior participants enjoyed the node applications that enabled them to preserve and share memories. Hence, they quickly identified the ScreenGram and Slideshow applications as a potential collective photoalbum, which could help preserve and rework memories, acting as a vehicle for their remembrance narratives. In addition, since the screen node is located within one of the social hubs in which our senior participants congregate, it can also serve as a focus point to talk about these memories. Hence, the visual elements of the screen facilitate the verbal storytelling with which they usually accompany photographs, and which they traditionally employ to build a sense of belonging.

Although seniors were not able to interact easily with the screen node (researchers helped them upload images and display them in the node), they enjoyed the social opportunities provided by using the node to share images in their community group. Rather than a superficial feeling derived from watching a large number of generic images, these participants preferred to dwell for longer in specific images that held importance for lives of others in the neighborhood; they preferred the images that better allowed them to retain collective memories, rather than those that simply had aesthetic appeal. They stressed that they would have liked to have a pause button to dwell for longer on specific images and discuss them, rather than having an automatic timed loop. Capturing an image in a photograph, and re-capturing the moment by pausing its display on the screen node, enhances the process of building intimate connections with the place and with other people. Hence, for these users remembrance is less about simple archiving and more about reclaiming the experiences of others as own, and enabling their own experiences to be reclaimed in the same way by 
others. Hence, our senior participants expressed a need to discuss specific images that participants of other ages did not require, and which directly connected with their place building efforts. Screen nodes helped seniors share their place building perceptions with each other, reinforcing their commonalities and leading to a more rewarding sense of place identity based on collective participation and mutual reinforcement.

\section{Discussion and Conclusions}

When interacting with the screen nodes, most participants (irrespective of their age) enact performances that connect them to their existing place building practices. These performances consist of patterns of behavior "whose repetitions situate actors in time and space, structuring both individual and group identities" [9]. Although these performances are based on repetition, mimicry and reproduction of social interaction in the neighborhood, they are also varied: to an extent, each performance is unique, allowing locals the expression of individuality even when following normative patterns of interaction. On the one hand, the repetition associated with each performance serves to deeply embed patterns of interaction and produce a shared sense of place. On the other, the freedom that each performance affords the individual can become a vehicle for the exploration of the relationships between the individual and the community. Hence, these performances are both agents of social change and aids to reproduce current social structures of interaction.

Although anchoring, scaffolding and action based approaches are universal human experiences regardless of age, we found that the behavior of our participants towards the screen could be understood (albeit in a simplified manner) by considering one of these approaches as dominant. We posit that this simplification can provide useful guidance when designing applications for public interactive screens, particularly regarding their longer-term integration with the social dynamics of a locale.

In this paper, we emphasized the commonalities between regular place building practices and the performances that locals enacted when interacting with screen nodes. To understand this performance, simply conducting interviews and recording the spoken word is not enough, as it misses the sociological and cultural milieu in which the performance acquires its meaning and cultural significance. Hence, we argue that understanding the wider context through ethnography and workshops in the wild provide an invaluable tool to make sense of the ways in which technologies become embedded within the place building practices of a locale.

Place building is directed towards the generation of a structure of feeling [21]; a deep subconscious familiarity with our everyday living spaces. Hence, it is not a purely emotional construction: it is also physical, leading to a deep-seated bodily experience in which the residents belong in the space and are able to navigate it effortlessly. It depends not only on the values, beliefs and customs of locals; it also depends on the unique ways in which their pre-existing place building practices create a bridge between their culture and the locale in which they live. In this sense, place building takes the spatial analysis of interaction with users and extends it with cultural dimensions, making it a useful addition to the tools that can be applied when considering the deployment of a technology in a given public setting. 
Acknowledgements. This work is funded by the UK DE Program grant EP/I031839/1 and EP/I031413/1. We would like to specially thank our host venues and research participants.

\section{References}

1. Appadurai, A.: Modernity at Large: Cultural Dimensions of Globalization. Minnesota Press, USA (1996)

2. Behrens, M., et al.: Exploring the Effect of Spatial Layout on Mediated Urban Interactions. In: Proc. of the ACM Int. Symp. On Pervasive Displays, PerDis (2013)

3. Belk, R.W.: Possessions and the extended self. Jrnl. of Consumer Res., 139-168 (1988)

4. Dant, T., Deacon, A.: Hostels to homes: The rehousing of single homeless people. Avebury/Gower, Aldershot (1989)

5. Dourish, P.: Re-space-ing place: 'place' and 'space' ten years on. In: Proc. of CSCW, November 4-8 (2006)

6. Gen, F., Schieck, A., et al.: Research in the Wild: Exploring the potential of Networked Urban Screens for Communities and Culture. In: DIS Workshop: Research in the Wild, Newcastle, UK (2012)

7. Giddens, A.: The Construction of Society. Polity, Cambridge (1984)

8. Greenfield, P.: A Theory of the Teacher in the Learning Activities of Everyday Life. In Everyday Cognition, pp. 117-138. Harvard University Press (1984)

9. Kapchan, D.: Performance. Journal of American Folklore 108, 479 (1995)

10. Memarovic, N., et al.: Using public displays to stimulate passive engagement, active engagement, and discovery in public spaces. In: Proc. of MAB (2012)

11. Miller, D.: Tales from Facebook. Polity (2011)

12. North, S., et al.: Tension Space Analysis: Exploring Community Requirements for Networked Urban Screens. In: Proceedings of IFIP INTERACT. LNCS. Springer (2013)

13. O'Hara, K., et al.: Understanding collective play in an urban screen game. In: Proc. CSCW 2008, San Diego, CA, USA (2008)

14. Redhead, F., et al.: NNUB: the neighbourhood nub digital noticeboard system. In: Proc. of ACM OzCHI, pp. 418-419 (2010)

15. Peltonen, P., et al.: It's Mine, Don't Touch!: interactions at a large multi-touch display in a city centre. In: Proc. of ACM SIGGHI (2008)

16. Satchell, C., et al.: Suburban nostalgia: the community building potential of urban screens. In: Proc. of ACM OzCHI (2008)

17. Taylor, K.: Programming video art for urban screens in public space. First Monday, Special Issue \#4: Urban Screens (2006)

18. Taylor, N., et al.: Social interaction around a rural community photo display. International Journal of Human-Computer Studies 67(12), 1037-1047 (2009)

19. Vertovec, S.: Towards post-multiculturalism? Changing communities, conditions and contexts of diversity. International Social Science Journal 61(199), 83-95 (2010)

20. Warnier, J.P.: A praxeological approach to subjectivation in a material world. Journal of Material Culture 6(1), 5-24 (2001)

21. Williams, R.: The Long Revolution. Pelican, Harmondsworth $(1961,1984)$

22. (2011), http://www.ons.gov.uk/ons/guide-method/census / 2011 / index.html 